\title{
Structure and function of the normal myocardium $^{1}$
}

\author{
Eugene Braunwald \\ From the Department of Medicine, University of California, San Diego, California, U.S.A.
}

The myocardium is composed of individual striated muscle cells (fibres), Io to $15 \mu \mathrm{m}$. in diameter and 30 to $60 \mu \mathrm{m}$. in length. Under the light microscope each fibre contains multiple cross-banded strands (myofibrils), which run the length of the fibre and are composed of a serially repeating structure, the sarcomere. The remainder of the cytoplasm, lying between the myofibrils, contains other cell constituents such as the single centrally located nucleus, numerous mitochondria, and intracellular membrane systems.

The sarcomere, the fundamental structural and functional unit of contraction, is delimited by two adjacent dark lines, the $Z$ lines (Fig. I). The distance between $Z$ lines varies with the degree of contraction or stretch of the muscle and ranges between $\mathrm{I} \cdot 5$ and $2.2 \mu \mathrm{m}$. Within the confines of the sarcomere alternating light and dark bands are seen, giving the myocardial fibres their striated appearance under the light microscope. At the centre of the sarcomere is a broad dark band of constant width ( $1 \cdot 5 \mu \mathrm{m}$.), the A band, which is flanked by two lighter bands, the I bands, which are of variable width. The sarcomere of heart muscle, like that of skeletal muscle, is made up of two sets of myofilaments. Thicker myofilaments, composed of the protein myosin, traverse and are limited to the $\mathrm{A}$ band. The myosin filaments are about $100 \AA$ in diameter, with tapered ends, and they measure $\mathrm{I} \cdot 5$ to $\mathrm{I} \cdot 6 \mu \mathrm{m}$. in length. Thinner myofilaments, composed primarily of actin, course from the $Z$ line through the $I$ band into the $\mathrm{A}$ band. The thin filaments are approximately $50 \AA$ in diameter and $\mathrm{r} \cdot 0 \mu \mathrm{m}$. in length. Thus there is overlapping of thick and thin filaments only within the A band, while the I band contains only thin filaments (Fig. I). Bridges extend between the myosin and actin filaments within the $A$ band.

The 'sliding' model for muscle rests on the fundamental observation that both the thick and thin contractile filaments are constant in

\footnotetext{
${ }^{1}$ Research supported in part by Public Health Service Grant.
}

overall length, both at rest and during contraction. With activation of the sarcomere, an interaction takes place between specific complementary sites on the actin and myosin filaments, and the actin filaments are propelled further into the $A$ band. In the process the $\mathrm{A}$ band remains constant in width, whereas the $I$ bands become more narrow and the $Z$ lines move toward one another (Huxley, 1969).

The sarcoplasmic reticulum, a complex network of anastomosing, membrane-lined intracellular channels which invests the myofibrils, has two distinct components. One portion, the longitudinal component, consists of a series of interconnecting membrane tubules closely applied to the surfaces of the individual sarcomeres and has no direct continuity with the outside of the cell. The second component, the transverse component or ' $\mathrm{T}$ ' system, is formed by tubelike invaginations of the sarcolemma, which extend into the myocardial fibre along the $\mathrm{Z}$ lines - that is, the ends of the sarcomeres.

There is evidence that once the excitatory stimulus, in the form of an electrical depolarization of the cell membrane, reaches the interior of the fibre via the transverse tubular system, it is transferred to the adjacent terminal cisternae of the longitudinal system, and $\mathrm{Ca}^{++}$which is contained therein is released from the sarcoplasmic reticulum. The $\mathrm{Ca}^{++}$diffuses into the myofibril and activates the myofilaments to produce contraction. The sarcoplasmic reticulum then appears to reaccumulate $\mathrm{Ca}^{++}$, thereby lowering its concentration in the myofibril to a level that inhibits the actin-myosin interaction which is responsible for contraction and in this manner leads to relaxation. Thus the sarcoplasmic reticulum, with its ability to transmit an action potential, to release and then reaccumulate $\mathrm{Ca}^{++}$, appears to play a fundamental part in the rhythmic contraction and relaxation of heart muscle (Davies, 1963).

In all forms of striated muscle, including cardiac muscle, the force of contraction depends on initial muscle length. The sarcomere 
SARCOMERE

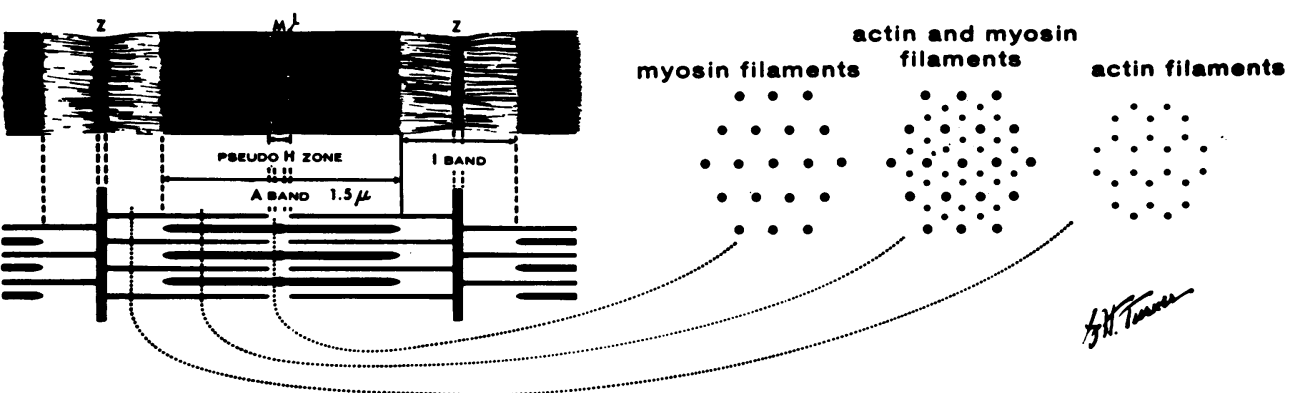

FIG. I (Left) A diagrammatic representation of the arrangement of myofilaments that make up an individual sarcomere from a myofibril, with thick filaments $(I \cdot 5 \mu m$. in length, composed of myosin) forming the $A$ band, and thin filaments ( $I \cdot 0 \mu \mathrm{m}$. in length, composed primarily of actin) extending from the $Z$ line through the $I$ band into the $A$ band, and with overlapping of thick and thin filaments seen only in the A band. (Right) Cross sections of the sarcomere indicate the specific lattice arrangements of the myofilaments. In the centre of the sarcomere, left, only the thick, or myosin, filaments arranged in a hexagonal array are seen; in the distal portions of the $A$ band, centre, both thick and thin, or actin, filaments are found, with each thick filament surrounded by six thin filaments; and in the I band, right, only thin filaments are present. (From Braunwald, Ross, and Sonnenblick, 1968).

length associated with the most forceful contraction is $2.2 \mu \mathrm{m}$. It is at this length that the two sets of myofilaments of the sarcomere are most ideally situated to provide the greatest area for their interaction. In support of the sliding-filament hypothesis, force development diminishes in direct proportion to the decrease in the overlap between thick and thin filaments and the resultant decrease in the number of reactive sites. At a sarcomere length of $3.65 \mu \mathrm{m}$. developed tension falls to zero, and it is at this point that the thin filaments are entirely withdrawn from the A band. Similarly, when the sarcomeres are shorter than $2.0 \mu \mathrm{m}$. the thin filaments bypass one another, producing a double overlap of the thin filaments, a less-than-ideal arrangement for interaction between thick and thin filaments.

The relation between the initial length of the muscle fibres and the developed force is of prime importance for the function of heart muscle. This forms the basis of the FrankStarling relation (Starling's law of the heart), which states that, within limits, an augmentation of initial volume of the ventricle, which is a function of the initial length of the muscle, results in an increase in the force of ventricular contraction. It has been shown for heart muscle, as well as for skeletal muscle, that sarcomere length is directly proportional to muscle length along the ascending limb of the length-active tension curve. As muscle length decreases to the point at which developed tension approaches zero and at which sarcomere length approaches $\mathrm{I} \cdot 5 \mu \mathrm{m}$., the I bands at first narrow, then disappear, while the $A$ band remains constant in length. At this latter point the $\mathrm{Z}$ line abuts on the edges of the $\mathrm{A}$ band. From these considerations it is apparent that the sarcomere length-active tension curve forms the ultrastructural basis of Starling's law of the heart. It is noteworthy that these points concerning the mechanism of contraction of the heart muscle fibre are also applicable to the skeletal muscle fibre.

\section{Myocardial mechanics}

Extremely helpful methods for examining the behaviour of muscle were provided by the skeletal muscle physiologists early in this century. The mechanical activity of all muscle may be expressed externally in only two ways: shortening and the development of tension. Hill (1938) showed in skeletal muscle that the velocity of shortening is inversely related to the magnitude of tension development, an expression of the so-called force-velocity relation, now acknowledged to be a fundamental property of muscle. Expressed simply, the greater the load the muscle is called upon to lift, the slower the velocity of shortening and vice versa. More recently the concept of the force-velocity relation has been extended from skeletal to cardiac muscle. The contractile 
activity of the myocardium may be readily altered under physiologic conditions by changes in resting fibre length and by changes in contractility, both of which shift the myocardial force-velocity curve (Sonnenblick, 1962).

All variations in myocardial contractile activity can be expressed as displacements of the force-velocity curve. However, there are two fundamental ways in which the forcevelocity curve can be shifted. Fig. 2 shows a family of force-velocity curves obtained from an isolated cardiac muscle; each curve was obtained at a different preload - that is, with a different degree of stretch on the muscle. Note that changing the preload has altered the intercept of the force-velocity curve on the horizontal axis - that is, it has increased the isometric force developed by the muscle. However, these alterations in preload have not altered the intrinsic velocity of shortening, since all the curves extrapolate to the same intercept on the vertical axis. Thus, a change in initial length of heart muscle shifts the force-velocity curve by altering the total force which can be developed by the muscle.

This type of shift in the force-velocity curve may be contrasted with that obtained when a positive inotropic agent, such as norepinephrine or digitalis, is added to the muscle while the initial length is held constant (Fig. 3). These agents not only increase the force which the muscle is capable of lifting, the intercept of the force-velocity curve on the horizontal axis, but also increase the velocity of shortening of the unloaded muscle, the extrapolated intercept on the vertical axis.

It has been postulated that an increase in initial muscle length brings about an increase in the number of force-generating sites operating effectively without any alteration in the qualitative character of the cyclic process at these contractile sites. Such a change in the contractile state, characterized by an increase in the velocity of shortening of the unloaded muscle, appears to result from an increase in the rate of cyclic force-generating processes at the contractile sites, without a change in the number of these sites (Sonnenblick, 1962).

\section{Contraction of the intact ventricle}

It was shown clearly in the heart-lung preparation that the stroke volume is a function of diastolic fibre length, and that the failing heart delivers a smaller-than-normal stroke volume from a normal or elevated enddiastolic volume. Later, the concept of measuring stroke work (the product of stroke vo't me and mean aortic pressure) over a range of mean atrial or ventricular end-diastolic

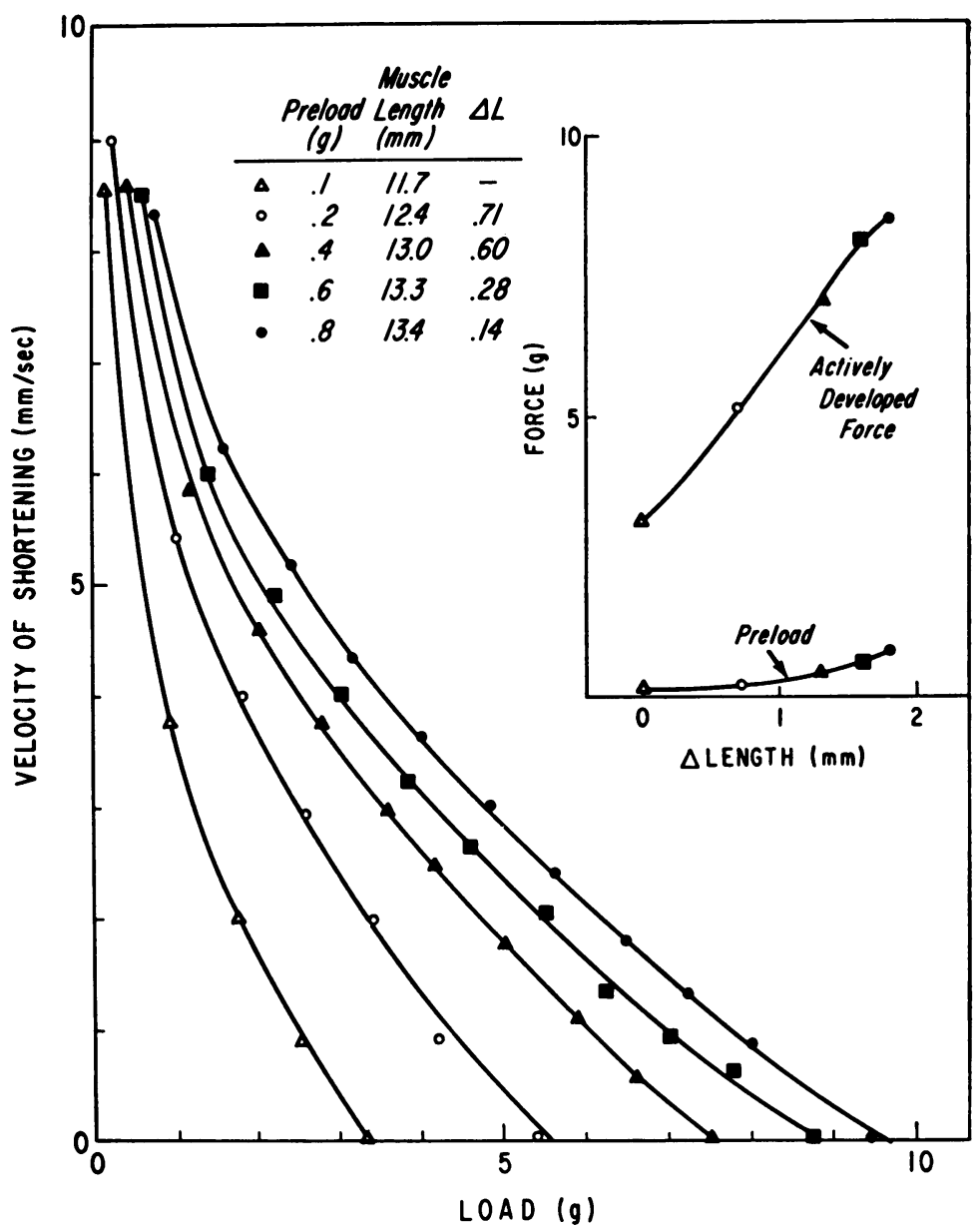

FIG. 2 Effects of increasing initial muscle length on the force-velocity relation of the cat papillary muscle. Initial velocity of shortening has been plotted as a function of load for five different muscle lengths. The maximum velocity of shortening $\left(V_{\max }\right)$ is essentially unchanged, whereas the maximum force of contraction $\left(P_{0}\right)$ is augmented. The insert shows the places along the length-tension curves at which these forcevelocity curves were determined. (From Braunwald et al., 1968.)

pressures, using one of these pressures as an index of diastolic volume, was expanded by Sarnoff and Berglund (1954).! They concluded that this relation between the mean atrial or the ventricular end-diastolic pressure and the stroke work of the corresponding ventricle (the ventricular function curve) provided a definition of the level of the contractile state of the ventricle. Significant increases in the level of ventricular contractility were accompanied by shifts of the ventricular func- 


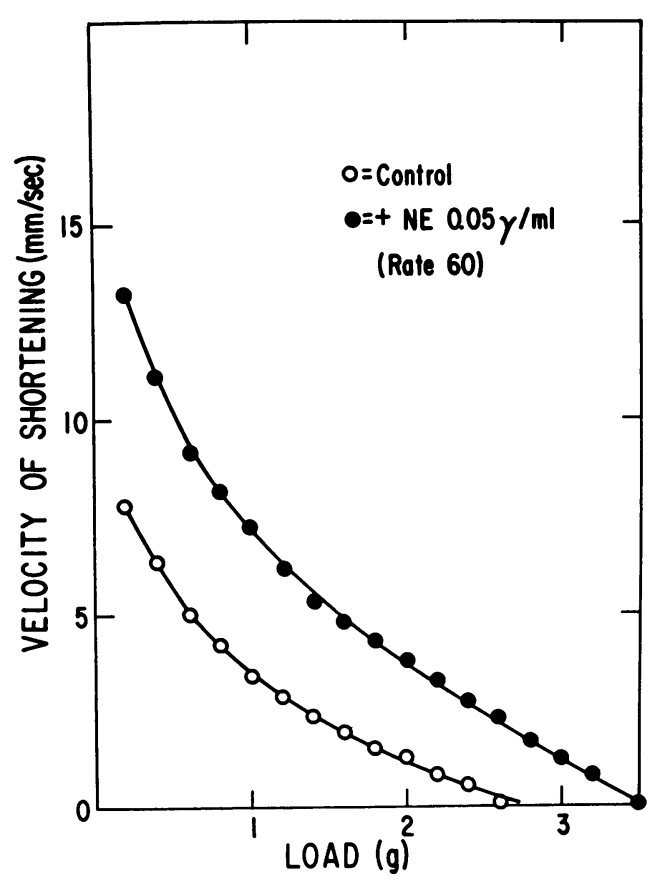

FIG. 3 Effects of norepinephrine on the forcevelocity relation of the cat papillary muscle. Both $V_{\max }$ and $P_{0}$ have been increased. (From Braunwald et al., 1968.)

tion curve upwards and to the left, while depression of contractility was identified by downward and rightward displacement of this relation (Fig. 4).

Recently considerable effort has been directed toward a study of the responses of the intact unanaesthetized animal, and it has been observed that during the adrenergic stimulation of the myocardium accompanying a stress such as exercise, no change or an actual decrease in ventricular end-diastolic size occurs while minute cardiac output, aortic flow velocity, and the rate of ventricular pressure development are augmented. Thus reflex and humorally mediated changes in myocardial contractility, heart rate, venous return, and peripheral vascular resistance often appear to overshadow the effects of the intrinsic FrankStarling mechanism - that is, the relation between end-diastolic pressure or volume and stroke work.

The important influence of the neurotransmitter substance norepinephrine on the mechanical and electrical properties of the myocardium has long been recognized. Direct stimulation of the stellate ganglions and reflex stimulation of the heart consequent to hypotension in the carotid sinuses have been shown

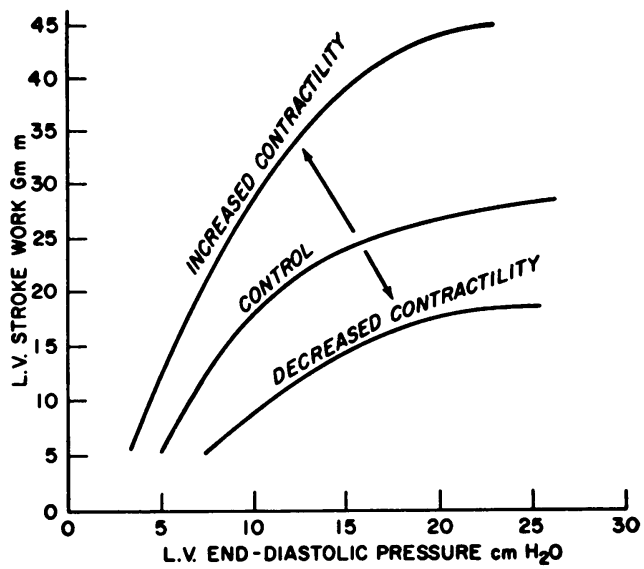

FIG. 4 Diagrammatic representation of ventricular function curves obtained under control conditions during the administration of a positive inotropic agent (increased contractility) and during a negative inotropic state (decreased contractility). $L V$, left ventricular. (From Braunwald et al., 1968.)

to elevate the ventricular function curve, as a consequence of the release of norepinephrine from sympathetic nerve endings in the heart. In the intact animal, these adrenergic effects are evidenced by tachycardia, a reduction in cardiac dimensions, increased velocity of ejection, and an enhanced rate of tension development. Cerebral ischaemia also appears to cause a profound increase in ventricular performance.

Surgically denervated hearts in situ, or isolated papillary muscles taken from such hearts, do not exhibit depression of their intrinsic contractile properties despite depletion of the norepinephrine stores in the sympathetic nerve endings of the muscle. The denervated heart of the intact animal also appears capable of meeting many of the demands of muscular exercise. However, the mechanisms by which the denervated heart increases its output differ from those of the intact animal. Thus tachycardia is less marked, and in animals subjected both to denervation and adrenalectomy the stroke volume and cardiac output rise as a consequence of elevation of ventricular enddiastolic volume. In all these respects the activity of the heart muscle fibre differs from the activity of skeletal muscle fibre, the latter being totally dependent on its nerve supply and unable to contract effectively when denervated. 


\section{Control of cardiac performance and car- diac output}

The extent of shortening of mammalian heart muscle and therefore the stroke volume of the intact ventricle are in the final analysis determined by three influences: (I) the length of the muscle at the onset of contraction, the preload; (2) the tension which the muscle is called upon to develop during contraction, the afterload; and (3) the contractile state of the muscle, the position of its force-velocitylength curve (Ross et al., I966).

At any level of its contractile state the performance of the myocardium is influenced profoundly by ventricular end-diastolic fibre length and therefore by diastolic ventricular volume. The following are the major determinants of ventricular end-diastolic volume in the intact organism: (I) total blood volume; (2) distribution of blood volume (body position, intrathoracic pressure, intrapericardial pressure, venous tone, pumping action of skeletal muscle); (3) atrial contribution to ventricular filling.

\section{Myocardial contractility}

A number of factors determine the level of ventricular performance at any given ventricular end-diastolic volume. These influences may be considered to operate by modifying the myocardial force-velocity-length relations: sympathetic nerve activity, circulating catecholamines, the force-frequency relation, exogenously administered inotropic agents, physiologic depressants, pharmacologic depressants, loss of ventricular substance, and intrinsic myocardial depression (Braunwald et al., 1969).

\section{Ventricular afterload}

The volume of blood ejected by the ventricle during each contraction is ultimately a function of the extent of ventricular fibre shortening during systole. The extent of shortening at any given level of diastolic fibre length and myocardial contractile state is inversely related to the afterload imposed on the muscle. The afterload on the intact heart is dependent on the level of aortic pressure, but it may be defined as the tension or stress developed in the wall of the ventricle during ejection. Therefore the afterload on the ventricular muscle fibres also is dependent on the size of the heart, according to the Laplace principle, which indicates that the tension of the myocardial fibre is a function of the product of intracavitary ventricular pressure and the ventricular radius. Thus, at the same level of aortic pressure, the afterload faced during systole by an enlarged left ventricle is higher than that encountered by a ventricle of normal size. The aortic pressure, in turn, is influenced largely by the peripheral vascular resistance, the physical characteristics of the arterial tree, and the volume of blood it contains at the onset of ejection. At any given ventricular end-diastolic volume and level of the myocardial contractile state, the left ventricular stroke volume is a function of the afterload.

All the influences acting on cardiac performance enumerated above interact in a complex fashion to maintain cardiac output at a level appropriate to the requirements of the metabolizing tissues, and in a normal individual interference with one or even a few of these mechanisms may not influence the cardiac output. For example, a moderate reduction of blood volume or the loss of the atrial contribution to ventricular contraction can ordinarily be sustained without a reduction in cardiac output. Presumably other factors, such as an increase in the frequency of sympathetic nerve impulses reaching the heart, will in the normal individual augment contractility and sustain output under these circumstances. Mechanisms are also available which prevent elevation of the cardiac output when there is no physiologic demand for augmented flow. For example, expansion of blood volume or augmentation of myocardial contractility by means of cardiac glycosides does not increase the cardiac output in normal man. Thus, in analysing the effect of an intervention on cardiac output, it is important to recognize that the contractile state of the myocardium is not the factor that limits the volume of blood ejected by the heart in the normal individual and that an improvement of myocardial contractility by a drug which exerts a positive inotropic effect (i.e. improves contractility), such as digitalis, or by a positive inotropic influence, such as paired electrical stimulation, should not be expected to elevate the output in a normal subject. On the other hand, in the presence of congestive heart failure the cardiac output usually is limited by the depressed contractile state of the myocardium, and a positive inotropic influence would be expected to raise cardiac output.

\section{References}

Braunwald, E., Ross, J., Jr., Gault, J. H., Mason, D. T., Mills, C., Gabe, I. T., and Epstein, S. E. (1969). Assessment of cardiac function. Annals of Internal Medicine, 70, 369.

,-- , and Sonnenblick, E. H. (1968). Mechanisms of Contraction of the Normal and Failing Heart. Little, Brown, Boston. 
Davies, R. E. (1963). A molecular theory of muscle contraction: calcium-dependent contractions with hydrogen bond formation plus ATP-dependent extensions of part of the myosin-actin cross-bridges. Nature (London), 199, 1068.

Hill, A. V. (1938). Heat of shortening and dynamic constants of muscle. Proceedings of the Royal Society, 126, 136.

Huxley, H. E. (1969). The mechanism of muscular contraction. Science, 164, 1356.

Ross, J., Jr., Covell, J. W., Sonnenblick, E. H., and
Braunwald, E. (I966). Contractile state of the heart characterized by force-velocity relations in variably afterloaded and isovolumic beats. Circulation $R e-$ search, 18, 149.

Sarnoff, S. J., and Berglund, E. (1954). Ventricular function. I. Starling's law of the heart studied by means of simultaneous right and left ventricular function curve in the dog. Circulation, 9, 706.

Sonnenblick, E. H. (1962). Force-velocity relations in mammalian heart muscle. American fournal of Physiology, 202, 931. 